\title{
Highly Efficient and Well-Matched Empty Substrate Integrated Waveguide H-plane Horn Antenna
}

\author{
Jorge Mateo, Ana M. Torres, Angel Belenguer, Senior Member, IEEE, and Alejandro L. Borja, Member, IEEE
}

\begin{abstract}
In this letter, an empty substrate integrated waveguide H-plane sectorial horn antenna is presented. The antenna is integrated within a planar substrate and is fed by means of a microstrip transmission line. It is composed of a partially empty substrate layer stacked between two cover metallic plates. Results obtained for a prototipe designed and fabricated at Ku-band show a measured efficiency close to $90 \%$ and matched responses. No extra loading elements are needed in order to reduce reflection at the end of the aperture conversely to previous designs. Moreover, the structure is quite simple, cheap and does not depend on substrate characteristics i.e. dielectric permitivity or loss tangent. This new methodology for manufacturing substrate integrated antennas without dielectric considerably improves the performance of conventional substrate integrated horn antennas.
\end{abstract}

Index Terms-Empty substrate integrated waveguide, substrate integrated waveguide, H-plane sectoral horn antenna, high efficiency.

\section{INTRODUCTION}

D URING the last years, a great number of substrate integrated circuits have been developed. These new circuits combine the advantages of classical non planar technologies (waveguide or coaxial transmission lines for instance) such as high quality factors, and the benefits of planar circuits such as low cost and easy compact integration. In this regard, substrate integrated waveguide (SIW) technology allows to construct several types of commonly used antennas in a planar way [1], [2]. However, different limitations associated to the use of the substrate appear in the implementation of certain types of antennas, i.e. low efficiency as frequency is increased due to dielectric loss, strong mismatch as a consequence of the use of thin substrate layers, and high reflection at the end of the aperture caused by the impedance difference between the dielectric and air. Several strategies have been developed to overcome these problems, and to optimize the design of dielectric-loaded horn antennas [3]-[7]. Some of them require the use of non-printed elements making the building procedure more complicated. Solutions based on integrated dielectric lenses have also been proposed but their performances are limited by the thickness of the substrate [8]-[12]. Nevertheless, the drawback related to losses introduced by the dielectric, and consequently low efficiency, has not been solved yet.

We present in this letter, a $H$-plane horn antenna with improved performance. The topology of the antenna is based

This work was supported by the Ministerio de Economía y Competitividad, Spanish Goverment, under Research Project TEC2013-47037-C05-3-R.

All authors are with Departamento de Ingeniería Eléctrica, Electrónica, Automática y Comunicaciones, Universidad de Castilla-La Mancha, Escuela Politécnica de Cuenca, Campus Universitario, 16071 Cuenca, Spain (email: jorge.mateo@uclm.es, ana.torres@uclm.es, angel.belenguer@uclm.es, alejandro.lucas@uclm.es. on the novel structure proposed in [13]. In this work, a new methodology for manufacturing empty substrate waveguides, without dielectric, but at the same time completely integrated in a planar substrate is proposed. The empty substrate integrated waveguide (ESIW) has an easy fabrication process while keeping the advantages of non planar structures such as high quality factor, quick simulation and optimization analisys and low loss. By this mean, horn integrated antennas with high radiation efficiency and good matched responses can be obtained.

The letter is structured as follows: Section II introduces the design and simulations of an ESIW horn antenna. Experimental results are discussed in Section III. Finally, Section IV is devoted to the conclusions of the paper.

\section{ESIW H-Plane Sectorial Horn Antenna Design}

The geometry of the ESIW H-plane sectorial horn antenna and the parameters used in the subsequent analysis are given in Fig. 1. As it can be seen, a $\lambda / 4$ adapter has been used to match the waveguide at $15 \mathrm{GHz}$. This ensures that almost all the energy that provides the microstrip line is transmitted to the horn antenna.

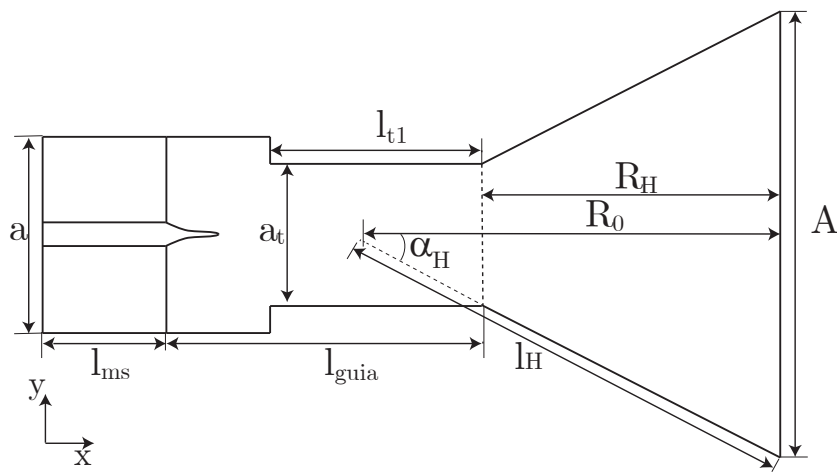

Fig. 1: Geometry of the ESIW $H$-plane sectorial horn antenna (x-y cut).

Next, the antenna design procedure followed is described. First, the size of the horn is chosen to obtain the desired directivity. In order to provide minimum phase error along the horn aperture the following expressions have been used [14]:

$$
l_{H}^{2}=R_{0}^{2}+\left(\frac{A}{2}\right)^{2},
$$




$$
\begin{gathered}
\alpha_{H}=\arctan \left(\frac{A}{2 R_{0}}\right)^{2}, \\
R_{H}=\left(A-a_{t}\right) \sqrt{\left(\frac{l_{H}}{A}\right)-\frac{1}{4},}
\end{gathered}
$$

where $A$ is the dimension of the horn antenna at the opening, $R_{H}$ is the radius of the horn and $l_{H}$ is the length of the antenna.

Then, a first version of the horn without transformer is analyzed. To do this, the horn antenna is directly fed by the guide of width $a$. In this step, the dimensions of the horn are calculated in terms of $a$, i.e. $a_{t}$ must be replaced by $a$ in (3). The results obtained serve to identify the resonances of the horn antenna. However, near the resonances, the field in the horn is distorted and the radiation pattern differs from the theoretical. As a result, it is more appropriate to match the horn between resonances, where the infield is stable and provides diagrams very similar to the theoretical radiation patterns.

Therefore, the horn is rescaled so that the identified resonances in the previous step are far from the design frequency. In this way, it must be ensured that the horn, with its new size, still fulfills the requirement of directivity. If this is not the case, the horn is rescaled again making it larger, but maintaining the resonances far from the design frequency. The next step is to analyze the horn antenna without transformer and estimate its input impedance. The reference plane to calculate the $S$ parameters is situated at the beginning of the horn and, in that case, its input impedance is:

$$
Z_{i n}=\frac{\left(1+S_{11}\right)}{\left(1-S_{11}\right)}
$$

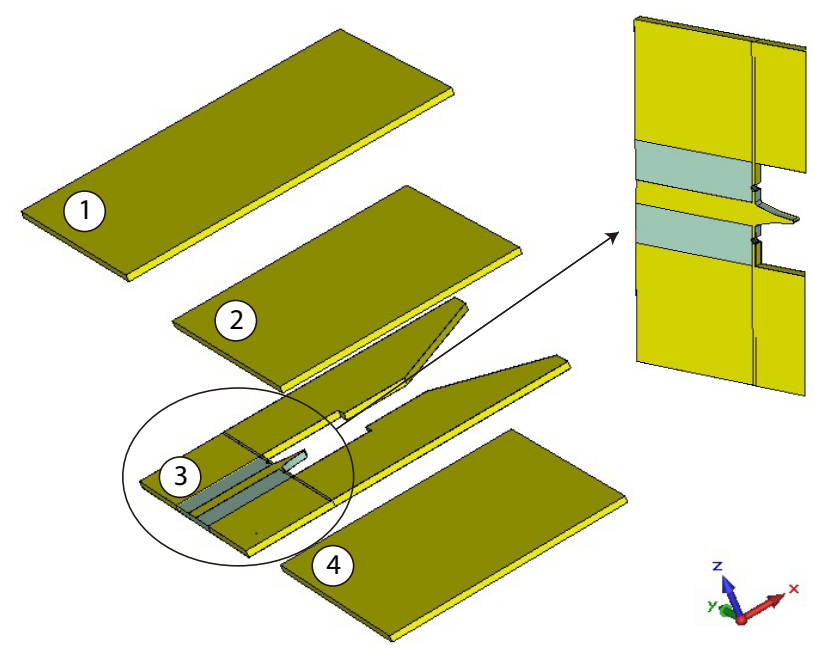

Fig. 2: ESIW layout (3D view).

The following is to design a transformer to adapt this impedance. In such a way $a_{t}$ and $l_{t 1}$ will be calculated. Finally the transformer dimensions and the radius of the horn antenna are optimized: $a_{t}, l_{t 1}$ and $R_{0}$. To make the horn antenna optimal, its dimensions are calculated using $a_{t}$, because now it is fed with that guide. The rectangular waveguide and sectorial horn antenna are integrated by using the same single substrate based on the ESIW technology (see Fig. 2). This figure is the model used for the simulation. This structure is designed by emptying an appropriate hole in a planar substrate which is closed with two thin upper and lower covers (numbers 2 and 4 in Fig.2). A secondary cover, on the top of the structure (number 1 in Fig.2) prevents undesired radiaton from the microstrip line and the SMA conector that feeds the antenna. The taper has been designed following the procedure highlighted in [13]. It is optimized with the following objetives $\left(S_{11}<-15 \mathrm{~dB}\right.$ and $\left.S_{21}>-2 \mathrm{~dB}\right)$ for a frequency range of $12 \mathrm{GHz}$ to $18 \mathrm{GHz}$. The final dimensions of the horn antenna are listed in Table I. In a traditional SIW, the electromagnetic waves are confined in a dielectric body enclosed by the upper and lower metallic walls of the substrate, and by lateral metallized circular holes. However, in the ESIW, the electromagnetic fields travel in the vacuum, and they are confined by upper, lower, and lateral metallic layers. Therefore the electromagnetic waves are propagated in the air, which implies low losses.

TABLE I: Parameters of the H-Plane Horn Antenna.

\begin{tabular}{lr}
\hline \hline Parameter & Value \\
\hline $\mathrm{a}(\mathrm{mm})$ & 15.79 \\
$\mathrm{a} t(\mathrm{~mm})$ & 10.53 \\
$\mathrm{l}_{t 1}(\mathrm{~mm})$ & 14.33 \\
$\mathrm{~A}(\mathrm{~mm})$ & 42.49 \\
$\mathrm{R}_{0}(\mathrm{~mm})$ & 30.118 \\
$\mathrm{R}_{H}(\mathrm{~mm})$ & 22.65 \\
$1_{\text {ms }}(\mathrm{mm})$ & 20 \\
$1_{\text {guia }}(\mathrm{mm})$ & 65 \\
\hline \hline
\end{tabular}

\section{EXPERIMENTAL AND SIMULATED RESULTS}

In order to validate the proposed design a prototype has been fabricated and measured. This structure is manufactured by emptying a hole in a planar substrate. Next, the substrate is metallized so that the lateral walls of the empty horn antenna are created. After that, the microstrip-to-ESIW taper is cut, and, finally, two thin upper and lower metallic walls are soldered to the substrate in order to confine the fields inside the horn. To fabricate this ESIW horn antenna, a LPKF Protomat S103 circuit board plotter was used. This machine provides a manufacturing resolution of $0.5 \mu \mathrm{m}$ and a repeatability of $\pm 0.001 \mathrm{~mm}$. The LPKF Mini Contac RS system was used for the electroplating. Fig. 3 shows the antenna prototype.

The commercial simulator Computer Simulation Technology (CST) Studio Suite 2014 has been used for the full-wave simulations. The simulated and measured reflection coefficient is shown in Fig. 4. As it can be seen, a good agreement is obtained between both responses. However, a small frequency 
shift of $41 \mathrm{MHz}$ is observed. Differences between simulation and measurements are due to the manufacturing process attributed to substrate permittivity variations, imperfections during the metallization and soldering and dimensions errors occurred during the milling. A narrow bandwidth around $268 \mathrm{MHZ}$ and $207 \mathrm{MHz}$ for simulations and measurements, respectively, is obtained. The best return loss is $50 \mathrm{~dB}$ at 15 $\mathrm{GHz}$ and $30 \mathrm{~dB}$ at $14.96 \mathrm{GHz}$ for simulated and measured results. Therefore, the antenna is well matched without use of external elements due to the absence of substrate.

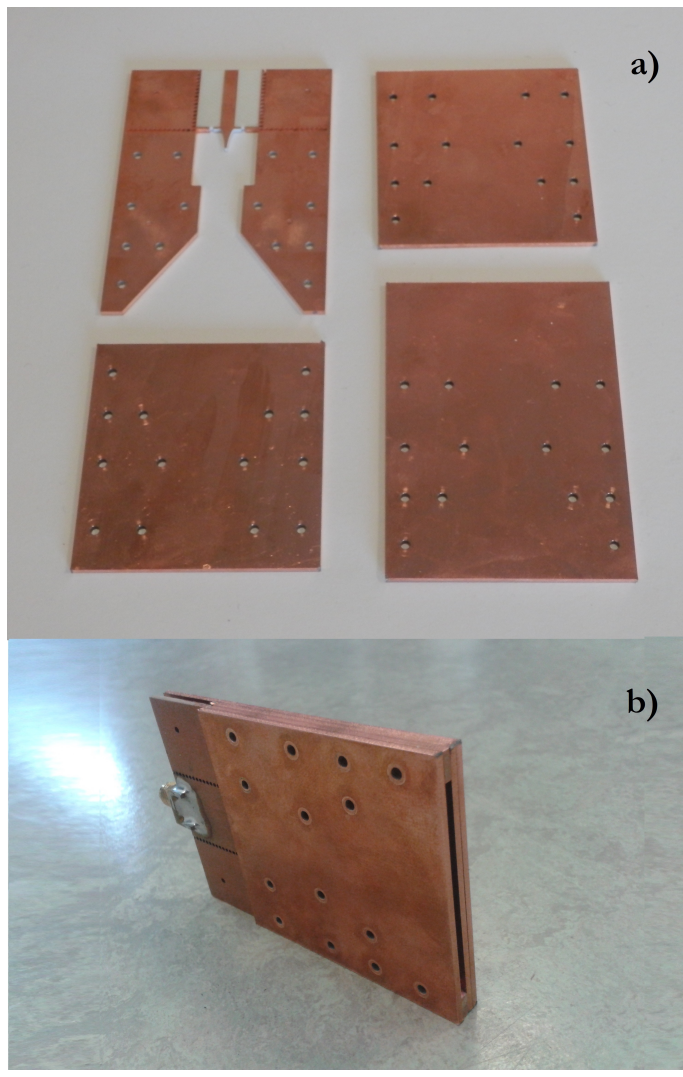

Fig. 3: a) ESIW $15 \mathrm{GHz} H$-plane sectoral horn antenna before assembling and b) Fabricated ESIW horn antenna prototype.

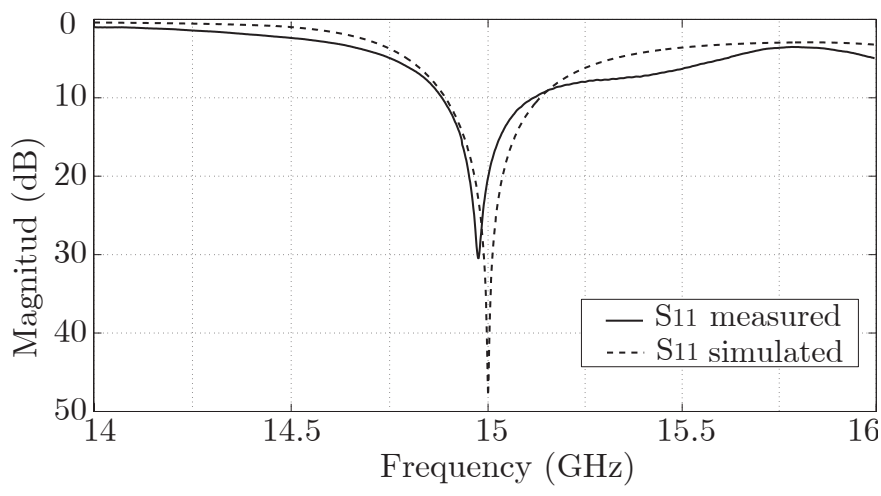

Fig. 4: Simulated and measured $|S 11|$ parameters.

The normalized 3D and E-plane radiation patterns are shown in Fig. 5. The radiation patterns have been obtained for the design frequency. A typical radiation pattern for H-plane sectorial horn antennas is obtained, see Fig. 5 a). However, some discrepancies can be observed due to the imperfections raised during the fabrication process. The most crucial point is the assembling of the different layers as a consequence of the generation of undesired resonances and air gaps inside the structure. Both effects can modify the radiation in certain directions and can also produce a strong ripple in the radiation pattern. In order to avoid the effects of these resonances and air gaps, a high quantity of soldering paste have been employed during the manufacturing of the antenna.
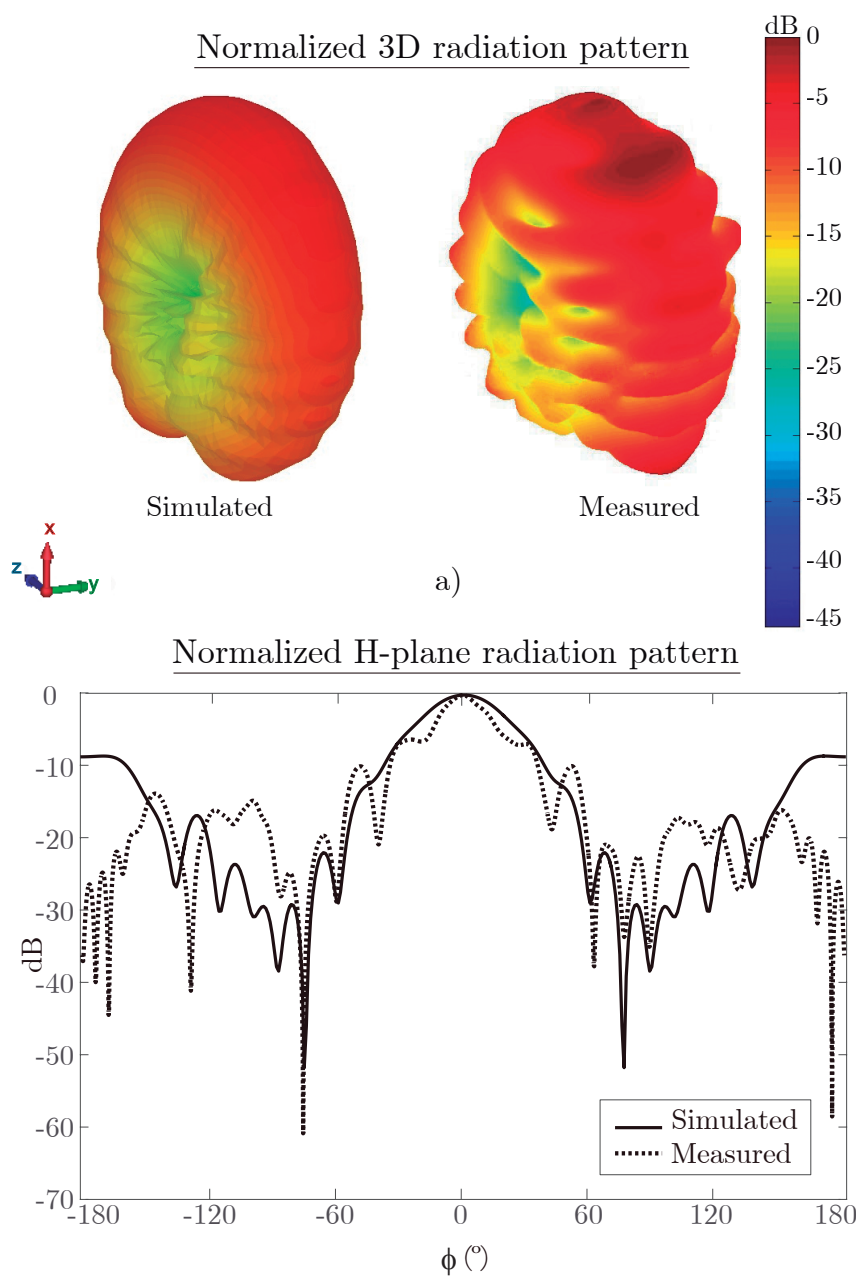

b)

Fig. 5: Normalized radiation pattern. a) 3D plot and b) E-plane plot.

The maximum of radiation is obtained along the $\mathrm{x}$-axis with measured directivity $8.94 \mathrm{dBi}$ and measured total efficiency $89.1 \%$. In Fig. 5 b), the maximum appears at $\theta=0^{\circ}$, as expected. Besides, the measured side lobe level (SLL) is 8.55 $\mathrm{dBi}$, the $-3 \mathrm{~dB}$ beamwidth and the beamwidth between first nulls are $15^{\circ}$ and $20^{\circ}$, respectively. In addition, an efficiency analysis including the values obtained for different ESIW and SIW antenna configurations, i.e. taking into account or not 
the micrsotrip feeding and SMA connector, has been carried out, see Table II. The simulated efficiency for the ESIW horn antenna including the microstrip feeding and the SMA connector is $92.9 \%$. The difference between measured and simulated results is due to quality of the electro-deposited copper that closes the structure. In the real device this metallic enclosure shows high degree of granularity while in simulations a perfectly plane copper layer has been considered. This fact increases the conduction losses in the metal, thus the efficiency is slightly worse (around 3.8\%) in experimental results. In order to compare the performance of this novel ESIW arrangement, an equivalent SIW horn antenna has been designed working at the same frequency. Both antennas have been simulated without considering the feeding lines connectors and transitions, so that only the target devices have been actually analyzed. The efficiency of the ESIW antenna is 99.9\% while the efficiency of the equivalent SIW antenna is only $69.3 \%$. A third part of the input power, i.e. a $30.6 \%$, is lost during the propagation inside the SIW antenna. Simulations also prove that approximately a $7 \%$ of the total radiation efficiency is lost when the feeding elements are considered (SMA connector, microstrip feeding line and transition to ESIW). Since, the measured efficiency for the whole antenna is $89.1 \%$, it is expected that the ESIW horn antenna itself will be able to provide a total radiation efficiency above $95 \%$.

Finally, it is worthwhile to mention that the high efficiency obtained for the proposed structure cannot be achieved with current SIW planar horn antennas due to substrate losses. This fact limit the use of substrate filled antennas to medium and low frequency ranges of operation.

TABLE II: Total efficiency for different ESIW and SIW antenna configurations.

\begin{tabular}{lc}
\hline \hline \multicolumn{1}{c}{ Antenna Configuration } & Total efficiency \\
\hline Measured ESIW with microstrip feeding and SMA & $89.1 \%$ \\
Simulated ESIW with microstrip feeding and SMA & $92.9 \%$ \\
Simulated ESIW with microstrip feeding & $94.8 \%$ \\
Simulated ESIW without microstrip feeding & $99.9 \%$ \\
Simulated SIW without microstrip feeding & $69.3 \%$ \\
\hline \hline
\end{tabular}

\section{CONCLUSiOnS}

An ESIW horn antenna with high efficiency and wellmatched is presented in this paper. This novel horn antenna has been designed and manufactured successfully. Simulated and experimental results show a radiation efficiency close to $89 \%$ and return losses around $30 \mathrm{~dB}$. The structure proposed improves the performance of previous SIW antennas. Potential use of these sort of antennas can be foreseen in a wide number of applications due to its easy integration and low cost.

\section{REFERENCES}

[1] Z. Li, K. Wu, and T. Denidni, "An new approach to integrated horn antenna," pp. 535-538, 2004.

[2] Z. Li, X.-P. Chen, and K. Wu, "A surface mountable pyramidal horn antenna and transition to substrate integrated waveguide," pp. 607-610, 2007.

[3] M. Esquius-Morote, B. Fuchs, J. Zurcher, and J. Mosig, "A printed transition for matching improvement of siw horn antennas," Antennas and Propagation, IEEE Transactions on, vol. 61, no. 4, pp. 1923-1930, 2013.
[4] J. Liu, D. R. Jackson, and Y. Long, "Substrate integrated waveguide (siw) leaky-wave antenna with transverse slots," Antennas and Propagation, IEEE Transactions on, vol. 60, no. 1, pp. 20-29, 2012.

[5] A. R. Mallahzadeh and S. Esfandiarpour, "Wideband h-plane horn antenna based on ridge substrate integrated waveguide (rsiw)," Antennas and Wireless Propagation Letters, IEEE, vol. 11, pp. 85-88, 2012.

[6] W. Che, B. Fu, P. Yao, Y. Chow, and E. K. Yung, "A compact substrate integrated waveguide h-plane horn antenna with dielectric arc lens," International Journal of RF and Microwave Computer-Aided Engineering, vol. 17, no. 5, pp. 473-479, 2007.

[7] L. Xue and V. Fusco, "Patch fed planar dielectric slab extended hemielliptical lens antenna," Antennas and Propagation, IEEE Transactions on, vol. 56, no. 3, pp. 661-666, 2008.

[8] H. Wang, D.-G. Fang, B. Zhang, and W.-Q. Che, "Dielectric loaded substrate integrated waveguide (siw)-plane horn antennas," Antennas and Propagation, IEEE Transactions on, vol. 58, no. 3, pp. 640-647, 2010.

[9] M. Bozzi, A. Georgiadis, and K. Wu, "Review of substrate-integrated waveguide circuits and antennas," Microwaves, Antennas \& Propagation, IET, vol. 5, no. 8, pp. 909-920, 2011.

[10] J. Yu, Y. Jiang, X. Lin, Z. Zhu, and Y. Fan, "A four-way half-mode siw power divider with improved impedance match," pp. 306-308, 2011.

[11] M. E. Morote, B. Fuchs, and R. J. Mosig, "Analytical model of a printed transition for siw antennas," in Proc. 6th EuCAP, pp. 414-417, March 2012.

[12] M. E. Morote, B. Fuchs, J.-F. Zurcher, and R. J. Mosig, "Novel thin and compact h-plane siw horn antenna," IEEE Trans. on Antennas, vol. 61, no. 6, pp. 2911-2920, June 2013.

[13] A. Belenguer, H. Esteban, and V. Boria, "Novel empty substrate integrated waveguide for high-performance microwave integrated circuits," Microwave Theory and Techniques, IEEE Transactions on, vol. 62, no. 4, pp. 832-839, April 2014.

[14] D. M. Pozar, Microwave engineering. John Wiley \& Sons, 2009. 